\title{
Le concept d'habitus chez Michel Henry
}

\section{Olivier Ducharme}

Journal of French and Francophone Philosophy - Revue de la philosophie française et de langue française, Vol XX, No 2 (2012) pp $42-56$

\author{
Vol XX, No 2 (2012) \\ ISSN 1936-6280 (print) \\ ISSN 2155-1162 (online) \\ DOI 10.5195/jffp.2012.521 \\ www.jffp.org
}

\section{(oc) EY-NC-ND}

This work is licensed under a Creative Commons Attribution-Noncommercial-No Derivative Works 3.0 United States License.

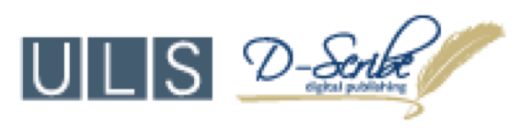

This journal is operated by the University Library System of the University of Pittsburgh as part of its D-Scribe Digital Publishing Program, and is co-sponsored by the University of Pittsburgh Press 


\title{
Le concept d'habitus chez Michel Henry
}

\author{
Olivier Ducharme
}

Univesité Laval

Michel Henry emploie à quelques occasions le concept $\mathrm{d}^{\prime}$ habitus ${ }^{1}$, mais sans lui donner une place particulière ou une importance quelconque. Par exemple, dans La barbarie, il traite de l'habitus en tant que pratique du corps effectuant le même mouvement. Effectuer un même geste jour après jour "détermine un habitus servant d'acquis et de substrat pour des effectuations ultérieures." ${ }^{2}$ Cette manière d'utiliser le concept d'habitus comme mouvement du corps fait immédiatement penser à l'article "Les techniques du corps" de Marcel Mauss. "La marche: habitus du corps debout en marchant, respiration, rythme de la marche, balancement des poings, des coudes, progression le tronc en avant du corps ou par avancement des deux côtés du corps alternativement (nous avons été habitués à avancer tout le corps d'un coup)." ${ }^{3}$ L'habitus se comprend ainsi comme une habitude du corps et se fonde, pour reprendre l'expression henryenne, "par la pratique et comme pratique." ${ }^{4}$ Dans un autre passage, Henry qualifie l'habitus de "typique" ou de "style." ${ }^{5}$ La pratique corporelle entendue comme mouvement, se comprend en tant que style dont use le corps pour se mouvoir.

Mais il faut également élargir la notion d'habitus comme style à tout ce qui a rapport avec notre manière de satisfaire nos besoins fondamentaux. Henry affirme: “Ainsi toute culture se définit-elle d'abord par les modalités selon lesquelles la vie répond à ses besoins, par des habitus concrets relatifs à la nourriture, au vêtement, au travail, à l'érotisme, à la relation à la mort, etc." ${ }^{6}$ Les habitus qui définissent la culture particulière d'une société se fondent tous, pour Henry, à partir de la pratique du corps. Nous percevons immédiatement que cette pratique du corps se lie profondément à une communauté culturelle. C'est par cette dernière que la pratique du corps se déploie à partir d'un "style" ou d'une "typique" particulière. Mais ici, il faut faire très attention. En admettant que la communauté culturelle est au fondement des différents "styles" d'habitus, il ne faut pas perdre de vue que

Journal of French and Francophone Philosophy | Revue de la philosophie française et de langue française Vol XX, No 2 (2012) | www.jffp.org | DOI 10.5195/jffp.2012.521 
ces différents styles se fondent eux-mêmes dans la pratique du corps. Pour Henry, ce qui est premier est la pratique et non pas la communauté culturelle. $^{7}$

Dès l'introduction de son premier ouvrage rédigé, Henry affirme la primauté de la pratique du corps subjectif par rapport aux différents "styles" d'habitus. Il écrit en note:

On dira, puisqu'il s'agit ici du corps, que, même si l'on admet notre réduction [réduction du corps objectif] et si l'on fait abstraction de toute évolution biologique en troisième personne, le corps humain s'offre à l'homme avec des caractères qui varient au long de l'histoire, caractères qui se traduisent par exemple dans les habitudes si diverses concernant la nourriture, le vêtement, la sexualité, ainsi que dans les nombreuses "modes" qui s'y rapportent. Il ne s'agit pas là, toutefois, du corps originel, mais des différentes façons pour l'homme de se représenter ce corps et de se comporter à son égard. Ce qui est historique, ce sont les objets culturels ou humains et les différentes attitudes humaines qui s'y rapportent. Mais le sol ontologique qui fonde les uns et les autres demeure indifférent à cette évolution; celle-ci présuppose toujours celui-là. ${ }^{8}$

Ce passage traduit la différence qui existe entre l'habitus et le fondement qui permet à cet habitus de se donner comme réalité. Mais de nouveau, il faut être prudent avec ce passage, car il semble bien que Henry situe l'habitus dans le monde de la "représentation," c'est-à-dire là où il est possible de créer une distance envers sa propre existence. Comme nous l'avons noté au sujet de l'habitus de la marche, il n'est pas question de mettre à distance notre manière de marcher et le fait que nous marchons réellement. Nous vivons réellement ce style de marche, nous expérimentons au plus près de soi le fait de marcher selon un certain habitus. Nous croyons que l'utilisation de Henry du concept de "représentation" vise plutôt à produire une différence entre le niveau originaire premier - le corps subjectif ou la pratique - et ce qui se greffe à cette origine - l'habitus.

La question de l'habitus se problématise encore davantage lorsque Henry élargit le champ de cette notion à notre appartenance à une nationalité ou à une ethnie. Il écrit dans un passage primordial de C'est moi la vérité:

Être grec ou juif, c'est se trouver déterminé sur le plan de la sensibilité, de l'affectivité, de l'intelligence, des modes de l'agir, subjectivement donc, selon des modalités vitales essentielles - et tout cela comme résultat de l'appartenance à une culture qui ne peut se définir elle-même que

Journal of French and Francophone Philosophy | Revue de la philosophie française et de langue française 
subjectivement, par des habitus fondamentaux de la vie transcendantale. ${ }^{9}$

Henry étend les limites de l'influence de l'habitus dans la vie de chaque vivant. Il ne s'agit plus uniquement de l'habitus de l'action du corps ou de la manière dont nous nous nourrissons, habillons ou travaillons. L'habitus se déploie à tous les niveaux de notre existence: la sensibilité, l'affectivité, l'intelligence du corps, etc. Être de telle ou telle nationalité, vivre dans tel ou tel milieu affecte profondément notre manière de vivre dans le monde, de sentir le monde, de bouger dans le monde, etc. Par cet élargissement de l'habitus, il est maintenant clair que nous ne pouvons plus l'entendre comme "représentation." Nous vivons sans distance ce que l'habitus nous porte à vivre. Il faut prêter une attention particulière à la manière dont Henry situe la portée de 1 ' "appartenance à une culture" à partir d' "habitus fondamentaux." Le fait d'être de nationalité grecque affecte fondamentalement la manière de l'individu de vivre et de sentir le monde. Être grec signifie, selon l'extrait cité, posséder une sensibilité et une affectivité propre au monde grec. Cette sensibilité et cette affectivité s'acquiert par certains "habitus fondamentaux" qui sont au fondement de l'identité affective d'une nation ou d'un peuple. En liant l'acquisition de certains "habitus fondamentaux" et de l'identité affective d'une nation, Henry nous pousse à croire que l'existence même de chaque nation ou de chaque communauté humaine se fonde sur la pratique du corps subjectif et ainsi sur l'habitus propre à cette pratique.

Devant une telle importance de la notion d'habitus, nous pouvons être étonnés de ne pas retrouver plus de développement au sujet de cette condition essentielle de la vie de chaque vivant. Henry s'en tient à ces quelques passages sans donner plus d'explication ou sans donner plus d'importance à cette notion. Les quelques explications que nous avons-nousmêmes données jusqu'ici ne suffisent pas à rendre compte des implications majeures que représentent une telle notion dans le champ de la phénoménologie henryenne. Ce que nous nous proposons de faire, dans cet article, est de situer la position du concept d'habitus dans la phénoménologie henryenne. Nous entrevoyons déjà une partie de la réponse à notre enquête grâce au passage cité de Philosophie et phénoménologie $d u$ corps: par la distinction entre le corps originel (sol ontologique) et le corps humain (corps historique subissant l'influence de l'habitus), Henry situe l'habitus au fondement de l'histoire, des objets culturels et du corps humain entendu comme objet culturel et historique. La distinction qui est ici en jeu entre le corps originel et le corps humain doit se comprendre à la lumière de la distinction qui se déploie dès l'introduction à L'essence de la manifestation entre l'être de l'ego et l'existence de l'ego vécu par un individu particulier. $C^{\prime}$ est à partir de cette distinction fondamentale, que nous retrouvons dans toute l'œuvre de Henry, que nous allons tenter de situer la place de l'habitus dans la phénoménologie matérielle.

Journal of French and Francophone Philosophy | Revue de la philosophie française et de langue française Vol XX, No 2 (2012) | http://www.jffp.org | DOI 10.5195/jffp.2012.521 


\section{L'être de l'ego et l'être de l'homme}

La problématique centrale de l'œuvre de Michel Henry a toujours été celle qu'il pose dès la première page de L'essence de la manifestation: "le sens de l'être de l'ego." ${ }^{10}$ La recherche henryenne se fonde ainsi sur une quête des principaux éléments qui forment l'être de l'ego, c'est-à-dire les conditions de possibilité de l'existence de ce dernier. En se basant sur cette recherche, Henry ne cherche pas à rendre compte uniquement de ces conditions comme si celles-ci formaient une région particulière de l'être, mais il cherche plutôt à montrer que cette problématique renvoie inévitablement à la question de l'être. ${ }^{11}$ Pour Henry, produire une phénoménologie de l'ego comme il le fait dans L'essence de la manifestation, c'est se questionner sur l'être. Comme il l'écrit dans une note préparatoire à ce dernier ouvrage: “L'Ego n'est pas un étant, mais l'être; il est l'être, et cela en un sens si radical qu'il nous est à peine permis de le penser." ${ }^{12}$ La question principale de l'introduction à L'essence de la manifestation porte précisément sur la définition de l'ego en tant que question de l'être comme en fait foi ce passage: "Ce qui est en question, bien au contraire, et cela d'une façon explicite, c'est la solidarité du sens de l'être de la réalité humaine avec celui de l'être en général."13 Henry cherche à montrer que la question de l'être ne peut se résoudre que si elle se pose à partir de l'ego lui-même. Toute l'œuvre de Michel Henry est une description de l'être en tant qu'ego.

Mais que faut-il entendre par "ego"? Quel sens lui donne Henry? Il faut immédiatement noter, avant de tenter de donner une réponse à cette question, que Henry s'est vite départi du concept d'ego et de "phénoménologie de l'Ego"; nous ne retrouvons plus ceux-ci dans les développements de L'essence de la manifestation, Henry emploie plutôt le concept de Soi. Dans un résumé analytique de L'essence de la manifestation rédigé en 1956-1957, Henry écrit: "Ce travail, entrepris en 1946, visait à l'origine la constitution d'une "Phénoménologie de l'Ego." L'introduction porte la trace de cette origine." ${ }^{14}$ Henry s'est servi de la question de l'ego comme point de départ à son travail, mais il l'a rapidement mis de côté. L'essence de la manifestation ne peut pas ainsi nous être d'une grande utilité, si nous recherchons une définition du concept d'ego. Il faut plutôt nous tourner vers Philosophie et phénoménologie du corps [texte rédigé en 1948-49], car cet ouvrage conserve encore la trace de cette "phénoménologie de l'ego" que tentait de produire Henry à cette époque.

Dans son commentaire de l'ontologie biranienne, Henry décrit ainsi l'être de l'ego:

Le moi [il faut lire ici "ego"], en effet, ne peut être compris comme la condition de toute connaissance que pour autant qu'il n'est pas "quelque chose" ni, comme nous l'avons dit, un étant, mais, précisément, la condition et 
l'élément même de la connaissance, l'élément ontologique de la manifestation pure. ${ }^{15}$

L'ego n'est pas cet étant particulier, il n'est pas cet étant-ci ou cet étant-là ou cet homme-ci ou cet homme-là, il est le fondement qui permet à ces singularités d'exister. Grégori Jean a très bien noté cette différence: “[...] l'Ego tel qu'il est nommé n'est pas le moi, n'est pas le Dasein, et n'est pas l'homme: "L'homme n'est pas l'ego, mais l'ego est l'essence de l'homme." Derrière les traits d'une "phénoménologie de l'ego," ce n'est donc pas d'une définition du "sujet" que Henry se met en quête, mais bien d'une saisie eidétique de l'être lui-même en tant qu'il possède une structure "subjective " [...]."16 Dans l'extrait que cite Grégori Jean, le concept $\mathrm{d}^{\prime \prime \prime}$ homme" représente l'existence singulière d'un homme vivant de telle ou telle manière dans le monde. La phénoménologie de l'ego que cherche à produire Henry ne vise pas à traiter de cet homme singulier vivant dans un milieu particulier, mais bien à mettre de l'avant les conditions de possibilité de l'existence de chacun de ces hommes singuliers. Une phénoménologie de l'ego va ainsi tenter de donner les "catégories"17, pour reprendre le vocabulaire de Philosophie et phénoménologie du corps, qui sont au fondement de l'être de l'ego. Henry cherche donc à établir "l'être de l'homme" lorsqu'il traite de l'ego comme le prouve cette note préparatoire à L'essence de la manifestation: “Certes, [le] Dasein n'est pas [1'] ego pour Heidegger, mais il désigne l'être de l'homme; or c'est ce que j'entends par ego."18

En abordant ainsi l'ego en tant qu'être de l'homme ne tombons-nous pas dans une recherche abstraite qui se fonde sur la quête d'une vérité impersonnelle? L'effort de Henry d'accorder la question de l'être à celle de l'ego nous force à admettre que l'être de l'homme n'a rien d'une vérité impersonnelle ou abstraite, mais qu'elle a tout à voir avec la vie même de l'ego. Pour Henry, nous devons lire "l'être de l'homme dans la subjectivité,"19 c'est-à-dire au cœur de la vie de chaque ego. Dans Philosophie et phénoménologie du corps, Henry spécifie encore plus la nature individuelle de l'ego en traitant de la question de l'essence de l'ipséité: “Ce qui constitue l'essence de l'ipséité, de l'égoïté [...], c'est l'intériorité de la présence immédiate à soi-même." ${ }^{20}$ Dans ce passage, Henry présente l'essence de l'ipséité en tant que synonyme de l'essence de l'ego, c'est-à-dire qu'il perçoit l'être (ou l'essence) de l'ego en tant qu'ipse, en tant que soi. Mais il faut immédiatement ajouter: un soi qui s'éprouve soi-même en tant que soi. C'est ce que Henry affirme lorsqu'il écrit que l'essence de l'ipséité est "l'intériorité de la présence immédiate à soi-même."

Henry conceptualise de manière encore plus précise l'essence de l'ipséité (ou de l'ego) dans le paragraphe 52 de L'essence de la manifestation. C'est dans ce paragraphe que Henry élabore le lien qui unit l'affectivité et l'ipséité. Par le concept d'affectivité, Henry décrit le "se sentir soi-même du soi." Il écrit à ce sujet: "Ce qui se sent soi-même, de telle manière qu'il n'est pas quelque chose qui se sent mais le fait de se sentir ainsi soi-même, de telle manière

Journal of French and Francophone Philosophy | Revue de la philosophie française et de langue française Vol XX, No 2 (2012) | http://www.jffp.org | DOI 10.5195/jffp.2012.521 
que son "quelque chose" est constitué par cela, se sentir soi-même, s'éprouver soimême, être affecté par soi, c'est là l'être et la possibilité du Soi." ${ }^{21}$ Être soi, c'est s'éprouver soi-même sans l'apport d'un affect extérieur au soi. Pour Henry, la meilleure manière de décrire ce "s'éprouver soi-même du soi" est de dire qu'il y a identité entre l'affectant et l'affecté. Il n'y a pas ici de rapport entre un objet et un sujet, entre ce qui est extérieur et ce qui est intérieur, mais il y a une seule chose, une seule identité: l'identité entre l'affectant et l'affecté: l'affectivité. "L'identité de l'affectant et de l'affecté réside et se réalise, trouve sa possibilité non théorique mais réelle, l'effectivité de son effectuation phénoménologique, dans l'affectivité. L'affectivité est ce qui met toute chose en relation avec soi et ainsi l'oppose à toute autre [...]." 22 Il faut, pour Henry, qu'il y ait l'affectivité (identité de l'affectant et de l'affecté) pour que nous ayons une expérience du monde et de manière encore plus fondamentale, une expérience de notre propre soi. L'affectivité est ce à partir de quoi nous sommes en tant que soi (ou tant qu'ego). Il écrit explicitement: "L'affectivité est l'essence de l'ipséité." ${ }^{23} \mathrm{Il}$ ajoute: "Parce que l'affectivité est l'essence de l'ipséité, tout sentiment est en tant que tel, comme sentiment de soi, un sentiment du Soi, laisse-être, révèle, constitue l'être de celui-ci." ${ }^{24}$

Henry traite du sentiment du Soi pour décrire l'être et la possibilité du Soi. Mais de quel sentiment est-il question? L'intérêt de cette question se trouve dans la réponse négative qu'apporte Henry. Le sentiment du Soi ne doit jamais se confondre avec l'expérience d'un sentiment particulier. Henry écrit à ce sujet:

Ce n'est jamais le contenu particulier d'un sentiment, la tonalité affective propre qui le différencie et l'isole de tout autre, qui peut faire de lui le sentiment d'un moi, celui-ci, précisément, n'est jamais le contenu particulier d'un sentiment particulier. Le contenu particulier d'un sentiment particulier s'identifie avec lui, le déterminant ici comme "haine" et là comme "amour," comme "béatitude," "tristesse" ou "désespoir"." Mais ce n'est pas en tant que haine ou en tant qu'amour, en tant que béatitude, tristesse ou désespoir, qu'un sentiment est celui d'un moi, du moi qu'il enferme en lui et révèle. A chacune de ces déterminations affectives, assurément, à chaque sentiment particulier, un Soi appartient. Il lui appartient en tant que ce sentiment est chaque fois et nécessairement le sentiment de soi, non en raison de son caractère particulier, mais en raison de son caractère affectif; il est le "se sentir soimême" qui vit en lui comme ce qui le rend possible, comme ce qui rend possible l'identité du sentiment et de son contenu, bref, son essence, l'affectivité en tant que telle. ${ }^{25}$

Ce passage nous permet de spécifier la nature du Soi (ou de l'ego) que Henry tente de décrire. Tout d'abord, il affirme que tout sentiment

Journal of French and Francophone Philosophy | Revue de la philosophie française et de langue française 
particulier se fonde sur un Soi qui joue le rôle de fondement: le sentiment particulier est le constitué tandis que le Soi est le constituant. Deuxièmement, le sentiment du Soi, le "se sentir soi-même," est l'essence ou l'être de ce Soi. Le sentiment de soi n'est ainsi aucun des sentiments particuliers, mais en même temps il se retrouve dans chacun de ceux-ci, c'est ce qui explique pourquoi nous pouvons dire qu'il est l'essence du Soi.

Cette manière de traiter le rapport entre l'affectivité et l'existence de sentiment particulier nous donne la chance de peut-être mieux comprendre la distinction que nous avons décrite un peu plus haut entre l'être de l'homme et l'homme. L'affectivité, que nous avons décrite en tant que sentiment de soi, est ce qui est au fondement de chaque Soi, elle est ainsi l'être de l'homme ou l'être de l'ego pour reprendre le vocabulaire présent dans l'introduction à L'essence de la manifestation. Tandis que l'existence particulière $d^{\prime}$ un sentiment se rapporte à l'existence singulière d'un homme qui trouve sa possibilité dans l'affectivité. Le travail phénoménologique que Henry entreprend avec L'essence de la manifestation et Philosophie et phénoménologie du corps le pousse à s'intéresser uniquement à la description de l'être (ou de l'essence) du Soi (ou de l'Ego), c'est-à-dire à l'affectivité. Comme le note Grégori Jean:

Or, comme son titre l'indique assez clairement, une phénoménologie de l'ego, de tel ou tel ego, [...] tel n'est justement pas le thème de L'essence de la manifestation. Ce n'est pas de ce qui constitue tel ou tel ego comme tel ou tel, celui de tel ou tel individu, que traite L'essence, mais de l'essence de l'ego $[\ldots] .^{26}$

En s'attardant à décrire l'essence de l'ego (ou l'être du soi), Henry vise à émettre les principales composantes de ce qu'il nomme le "Soi originel." ${ }^{27}$ À ce Soi originel, Henry oppose un Soi transcendant dont il déplore, en quelque sorte, l'absence dans L'essence de la manifestation: "La théorie de la constitution de ce Soi transcendant est une des tâches propres de la Phénoménologie de l'Ego. Elle n'a pu prendre place dans le cadre de ces recherches." ${ }^{28}$ La distinction entre un Soi originel et un Soi transcendant se rapporte à la distinction entre l'être de l'homme et l'homme singulier.

Le Soi transcendant représente l'existence individuelle d'un homme, c'est-à-dire ce qu'est un homme: de tel sexe, de telle nationalité, né dans tel milieu, etc. À la différence du Soi originel, le Soi transcendant se transforme historiquement, il se développe au contact de culture particulière rendant ainsi l'existence singulière de l'homme changeante selon le milieu dans lequel il est né. La distinction majeure entre le Soi transcendant et le Soi originel est que le premier est culturellement déterminé, tandis que le second est le fondement qui permet à tout Soi d'être culturellement déterminé. La "théorie de la constitution" du Soi transcendant dont Henry a en vue dans L'essence de la manifestation, mais qu'il ne produit pas, vise ainsi

Journal of French and Francophone Philosophy | Revue de la philosophie française et de langue française

Vol XX, No 2 (2012) | http://www.jffp.org | DOI 10.5195/jffp.2012.521 
la description de l'existence particulière d'un homme dans une culture donnée: comment un homme peut-il devenir un homme ou une femme, un grec ou un juif, un esclave ou un maître, pour reprendre les exemples que donne Paul dans l'Épîtres aux Galates et que Henry cite à son tour dans C'est moi la vérité. ${ }^{29}$

\section{Le tournant anthropologique}

Même si Henry souligne la présence d'une "théorie de la constitution" du Soi transcendant et qu'il déplore, en quelque sorte, le fait qu'il n'ait pas pu en traiter dans le cadre de L'essence de la manifestation, nous ne retrouverons pas plus cette étude dans ces ouvrages subséquents. Le Marx, par exemple, poursuit les analyses amorcées dans L'essence de la manifestation en approfondissant la description du Soi originel. Henry écrit dans l'introduction au Marx:

De même en est-il pour tous les concepts fondamentaux de Marx: concepts d'individu, d'histoire, de la praxis ellemême qui ne sert de sol aux développements ultérieurs qu'à partir du moment où [...] elle est pensée comme telle et comme la détermination la plus intérieure de l'être. ${ }^{30}$

Par l'étude de la praxis, Henry note la présence de l'action au cœur de la structure de l'être entendue comme Soi originel. Henry perçoit la praxis comme étant une catégorie qu'il qualifie d'absolue: "Absolue est la vérité en tant qu'elle réside dans la réalité de la vie, dans la praxis." ${ }^{31}$ La praxis est ainsi une catégorie absolue qui est fondement de l'existence singulière de chaque homme. Il en est de même pour les autres grandes catégories que nous retrouvons dans le Marx, par exemple le besoin et l'histoire.

Nous pouvons dire la même chose de La généalogie de la psychanalyse ${ }^{32}$, de La barbarie ${ }^{33}$, de Voir l'invisible ${ }^{34}$ et de Phénoménologie matérielle. ${ }^{35}$ Aucun de ces ouvrages ne traite explicitement du Soi transcendant. De manière étonnante, nous devons attendre la publication de C'est moi la vérité pour pouvoir enfin lire une analyse du Soi transcendant. Par la description de l'égoïsme transcendantal, Henry développe, dans C'est moi la vérité, une véritable phénoménologie de la constitution du Soi transcendant, en établissant les bases de l'existence du Soi dans le monde. Il faut ainsi attendre le "tournant théologique" pour être en mesure de retrouver la figure de l'homme dans la phénoménologie matérielle. Ce revirement de situation a tout pour surprendre, car pour la plupart des commentateurs qui ont critiqué le "tournant théologique" de Michel Henry, la trilogie chrétienne (C'est moi la vérité, Incarnation, Paroles du Christ) représente un passage vers un "hyper-transcendantalisme," pour reprendre l'expression de Rudolf Bernet, c'est-à-dire vers l'étude de ce qui n'a pratiquement plus de contact avec le monde ou l'empirie. Dans son article "Christianisme et 
phénoménologie," Bernet écrit, à propos de l'hyper-transcendantalisme de Henry:

L'hyper-transcendantalisme de M. Henry et sa conception théologique de la Vie ne conduit pas seulement à l'abandon du monde, mais aussi à une dévalorisation de tout ce qui fait la concrétude de la vie humaine. [...] $\mathrm{M}$. Henry se désintéresse de tout de ce qui relève de la contingence ou de la facticité de l'existence humaine, c'està-dire les différences sociales, culturelles, sexuelles etc., au nom de la condition de l'homme comme Fils de Dieu. ${ }^{36}$

La question que nous devons poser à partir d'un tel constat est la suivante: Henry ne s'en est-il pas toujours tenu à décrire l'être ou la Vie absolue pour reprendre le vocabulaire développé dans la trilogie? Faut-il attendre la publication de la trilogie, et plus particulièrement la publication de $C^{\prime} e s t$ moi la vérité, pour retrouver chez Henry une réflexion portant uniquement sur la Vie absolue? D'après les analyses que nous avons effectuées au sujet de la différence qu'instaure Henry entre l'existence d'un Soi originel et le Soi transcendant et de l'absence de toute description de la "constitution" du Soi transcendant, nous sommes portés à répondre que l'intérêt de Michel Henry a toujours été la description de l'être ou de la Vie absolue et qu'il a toujours laissé de côté l'existence des "différences sociales, culturelles, sexuelles, etc.." Une telle réponse doit-elle nous décourager à jamais d'amorcer une description de la constitution du Soi transcendant à partir des fondements de la phénoménologie henryenne? Nous croyons que non; et la présence du concept d'habitus va nous permettre de mieux comprendre la possibilité d'une telle description.

Paradoxalement, et comme nous l'avons déjà noté, il faut attendre la publication de C'est moi la vérité pour retrouver la figure de l'homme dans la phénoménologie henryenne. En traitant de l'égoïsme transcendantal et surtout en établissant une distinction entre la Vie absolue (auto-affection forte) et la condition de l'homme en tant que Fils de Dieu (auto-affection faible), Henry décrit enfin l'existence quotidienne de l'homme et s'attarde ainsi au Soi transcendant. C'est à partir de cette réflexion sur la condition de l'homme que Grégori Jean écrit à propos du fameux "tournant théologique" de Henry: "[...] la "philosophie du christianisme" de M. Henry est le lieu d'une méditation phénoménologique radicale non pas sur Dieu mais sur l'homme. De sorte que s'il est un "tournant" dans la trilogie, il s'agit d'un tournant tout autre que celui que l'on a voulu y voir: un tournant anthropologique $[\ldots] . .^{\prime 37}$

\section{Présence de l'habitus}

L'absence de développement de la constitution du Soi transcendant, dans la plus grande part de l'œuvre de Henry, a des conséquences importantes pour 
tous les autres concepts décrits par la phénoménologie matérielle. La distinction entre un Soi originel et un Soi transcendant représente le fondement à partir duquel tout se joue dans l'œuvre de Henry. Nous devons ainsi situer l'habitus d'après cette dichotomie originelle: l'habitus se lie à l'existence du Soi transcendant, c'est-à-dire à l'existence individuelle de chaque individu. Le Soi originel, quant à lui, se "constitue" sans l'apport de l'habitus, il est plutôt ce qui est au fondement de la possibilité et de l'existence de l'habitus qui est au cœur du Soi transcendant. Mais de quelle manière doit-on comprendre le rôle essentiel que joue l'habitus dans la constitution du Soi transcendant? Comme nous allons vite le constater, l'habitus est au fondement de l'identité culturelle, sexuelle et fonctionnelle du Soi transcendant. Henry traite de ces trois types d'identité dans un passage-clé de C'est moi la vérité, dans lequel il décrit le rôle central que joue l'habitus dans la vie sociale de chaque individu vivant dans le monde.

Les trois types d'identité que Henry traite dans C'est moi la vérité l'identité culturelle, l'identité fonctionnelle, l'identité sexuelle - se définissent toutes par l'habitus. À la page 313, Henry élabore les trois types d'identité en commençant par l'identité culturelle. Il écrit, dans un passage que nous avons déjà cité, mais dont nous ne pouvons pas sous-estimer l'importance pour notre propre analyse:

Être grec ou juif, c'est se trouver déterminé sur le plan de la sensibilité, de l'affectivité, de l'intelligence, des modes de l'agir, subjectivement donc, selon des modalités vitales essentielles - et tout cela comme résultat de l'appartenance à une culture qui ne peut se définir elle-même que subjectivement, par des habitus fondamentaux de la vie transcendantale. ${ }^{38}$

Il ajoute immédiatement, mais cette fois au sujet de l'identité fonctionnelle:

Être maître, de la même façon, qu'il s'agisse d'un maître du temps de Paul ou d'un patron d'aujourd'hui, d'un serviteur ou d'un ouvrier, c'est être façonné par des modalités concrètes de la praxis, laquelle en tant que praxis réelle, individuelle, subjective, n'est elle-même qu'une détermination de l'agir vivant. ${ }^{39}$

Finalement, il écrit à propos de l'identité sexuelle:

Être un homme enfin ou une femme, c'est tout autre chose que présenter un certain aspect extérieur, des propriétés naturelles [...]. Ici encore, ce qu'on dit être "naturel" ou "objectif" ne peut se définir qu'à partir d'un certain nombre d'expériences subjectives transcendantales comme par exemple le déploiement intérieur et vécu de la "sexualité" féminine $[\ldots]^{40}$ 
Ce que ces trois types d'identité ont en commun est que tous trois s'acquièrent grâce à une pratique - à une praxis, selon Henry - et que cette pratique devient elle-même le lieu d'une habitude. L'habitus doit ainsi se comprendre en tant que pratique servant à modeler l'identité culturelle, fonctionnelle et sexuelle.

Ce qui différence au moins deux de ces trois types d'habitus est la manière dont elle s'acquiert. L'identité culturelle et l'identité sexuelle se différencient de l'identité fonctionnelle en raison du fait qu'elles s'acquièrent passivement, tandis que l'identité fonctionnelle se constitue de manière active, c'est-à-dire par l'activité consciente de l'individu. ${ }^{41}$ Comprise en tant que praxis de l'affectivité, l'habitus se révèle être le fondement de la constitution de l'identité de tout individu singulier. Henry a souvent traité de la problématique de l'identité du soi en abordant la question de l'ipséité. Comme nous l'avons noté précédemment, l'ipséité se définit en tant que "se sentir soi-même" ou en tant que "sentiment de soi." Comme nous l'avons également noté, pour bien comprendre la nature de ce "sentiment de soi," nous devons le détacher de tout sentiment particulier propre à l'existence singulière de chaque individu et le poser à l'origine de l'existence de tous les sentiments particuliers. Pour Henry, l'individu s'identifie à lui-même grâce à l'existence de ce "sentiment de soi," c'est-à-dire grâce à la possibilité pour l'individu de "se sentir soi-même" en tant que soi. Henry s'intéresse ainsi à décrire l'identité originelle ou essentielle de chaque individu. Toute son œuvre, peut-on dire, porte principalement sur la description de cette identité originelle de chaque individu singulier. Mais qu'en est-il, dans ce cas, de l'identité qui se joue au niveau culturel, sexuel et fonctionnel? Si nous posons la question, c'est parce que Henry n'a jamais cru bon aborder cette problématique de manière aussi développée que celle de l'identité originelle. Il y a certes le passage de C'est moi la vérité dans lequel Henry traite explicitement de la problématique de l'identité culturelle, sexuelle et fonctionnelle, et dans lequel l'habitus joue un si grand rôle, mais ce passage demeure sans suite dans l'œuvre de Henry. Mais que doit-on penser de l'identité qui se joue au niveau du soi transcendant, c'est-à-dire de l'identité que l'individu acquiert par l'habitus? N'est-elle pas celle qui est à la base de notre vie sociale quotidienne et celle à partir de laquelle nous vivons notre propre appartenance à une culture ou un genre sexuel particulier?

La phénoménologie matérielle produite par Henry ne traite pas explicitement de cette problématique, mais elle laisse un espace ou un milieu pour que nous puissions en parler. Henry a ouvert le chemin en identifiant les limites transcendantales à partir desquelles se développe une phénoménologie matérielle; par ce travail, il a permis la fondation d'une phénoménologie qui se base sur la vie affective de l'individu, c'est-à-dire sur le sentiment du soi ou sur le se sentir soi-même. En nous interrogeant sur la formation identitaire du soi transcendant, nous travaillons à repousser les limites de la réflexion phénoménologique de Michel Henry en incluant dans

Journal of French and Francophone Philosophy | Revue de la philosophie française et de langue française Vol XX, No 2 (2012) | http://www.jffp.org | DOI 10.5195/jffp.2012.521 
celle-ci non pas uniquement la vie et la description du soi originel, mais également la vie égotique qui est au fondement des sentiments particuliers que nous ressentons lorsque nous vivons avec autrui.

1

Michel Henry, La barbarie (Paris: P.U.F., 2004), 109, 166; Michel Henry, C'est moi la vérité (Paris: Seuil, 1996), 313; Michel Henry, Phénoménologie de la vie IV: Sur l'éthique et la religion (Paris: P.U.F., 2004), 34.

2 Henry, La barbarie, 109.

3 Marcel Mauss, "Les techniques du corps," Sociologie et anthropologie (Paris: P.U.F., 2004), 380.

4 Henry, La barbarie, 109.

5 Henry, La barbarie, 166. Sur ce passage voir: Marc Maesschalck, Transformations de l'éthique (Bruxelles: P.I.E. Peter Lang, 2010), 175; Benoît Kanabus, Généalogie du concept d'Archi-Soi chez Michel Henry (Hidelsheim/Zürich/New York: Olms, 2011), 152. Il est intéressant de noter la présence chez Husserl d'une description de l'habitus en tant que style et en tant que "soubassement" de "toutes les activités et passivités" de l'ego: “Il y a sans doute un sens à parler d'une individualité en tant que style d'ensemble et habitus du sujet qui, en tant qu'unité globalement concordante, traverse tous les modes de comportement, toutes les activités et passivités et auquel contribue aussi constamment le soubassement psychique tout entier." Husserl, Ideen II: Recherches phénoménologiques pour la constitution, trad. E. Escoubas (Paris: P.U.F., 1982) 373. Pour la question de l'habitus chez Husserl, se référer: Dermot Moran, "Edmund Husserl's Phenomenology of Habituality and Habitus" Journal of the British Society for Phenomenology, vol. 42, no. 1 (2011): 53-77.

${ }_{7}^{6}$ Henry, Phénoménologie de la vie IV, 34.

7 La preuve de cette affirmation nous est donnée dans un passage du Marx: "Comme l'être, l'intersubjectivité trouve son essence originelle dans la praxis." Michel Henry, Marx II: Une philosophie de l'économie (Paris: Gallimard, 1976), 119. 8 Michel Henry, Philosophie et phénoménologie du corps (Paris: P.U.F., 1965), 6.

Henry, C'est moi la vérité, 313.

10 Henry, L'essence de la manifestation (Paris: P.U.F., 1963), 1.

11 Henry écrit dans l'introduction à L'essence de la manifestation: "Ce dont nous sommes maintenant en présence, c'est, par conséquent, l'ego absolu, le naturant originaire qui n'appartient pas à une région déterminée de l'être et qui ne saurait être correctement pensé par nous sous le titre de "région 
conscience," puisqu'il est, au contraire, ce qui confère à l'ensemble des régions le sens que l'être revêt chaque fois en elles" (35).

12 Michel Henry, "Notes préparatoires à L'essence de la manifestation: "La subjectivité," Revue internationale Michel Henry 3 (2012): 106.

13 Henry, L'essence de la manifestation, 46. Nous pourrions également citer ce passage: "Le problème de l'être de la subjectivité nous renvoie, inévitablement, au problème du sens de l'être en général" (35).

14 Michel Henry, “L'essence de la révélation: Résumé analytique” in Michel Henry, dir. J.-M. Brohm et J. Leclercq (Lausanne: L’Age d'homme, 2009), 55.

15 Henry, Philosophie et phénoménologie du corps, 56.

16 Grégori Jean, "La subjectivité, la vie, la mort," Revue internationale Michel Henry 3 (2012): 22. G. Jean cite “Notes préparatoires à L'essence de la manifestation: "La subjectivité," 95.

17 "Et c'est parce qu'elle est véritablement transcendantale, c'est-à-dire appuyée sur une sphère d'immanence absolue, que la déduction biranienne des catégories n'est pas véritablement une déduction, mais plutôt, comme nous le verrons, une simple lecture des caractères phénoménologiques de l'ego" (Henry, Philosophie et phénoménologie du corps, 31-32).

18 Henry, “Notes préparatoires à L'essence de la manifestation: "La subjectivité," 105. Henry emploie à six autres occasions cette expression "l'être de l'homme" dans ces mêmes notes: 142-147-153-167-189-212.

19 Henry, “Notes préparatoires à L'essence de la manifestation: “La subjectivité," 189.

20 Henry, Philosophie et phénoménologie du corps, 53.

21 Henry, L'essence de la manifestation, 581.

${ }_{23}^{22}$ Henry, L'essence de la manifestation, 581.

${ }_{24}^{23}$ Henry, L'essence de la manifestation, 581.

24 Henry, L'essence de la manifestation, 581.

25 Henry, L'essence de la manifestation, 581-582.

26 Grégori Jean, “De l'expérience métaphysique d'autrui à l'intersubjectivité en première personne," Revue internationale Michel Henry 2 (2011): 65. Il écrit également: "Mais qu'en est-il dès lors de l'homme lui-même, autrement dit de l'homme en tant qu'il ne se confond pas absolument avec l'infinité et l'absoluité de l'essence en lui - c'est-à-dire de l'homme en tant qu'il est fini? C'est justement la question que L'essence de la manifestation, tournée vers

7 la manifestation de l'essence, ne pose pas [...]" (69).

27 Henry, L'essence de la manifestation, 754.

28 Henry, L'essence de la manifestation, 754.

29 Henry, C'est moi la vérité, 310.

30 Michel Henry, Marx I: Une philosophie de la réalité (Paris: Gallimard, 1976) 27. 28.

31 Henry, Marx I, 470. 
32

L'enjeu encore une fois est de dévoiler une ontologie qui permet de mettre en place les catégories essentielles de la vie de chaque ego. Henry en vient à la toute fin de son ouvrage à traiter de la Potentialité comme "possibilité ontologique originelle" du mouvement du corps: "Si la puissance est décrite comme la possibilité de ces actes [du corps], alors c'est cette possibilité à son tour qui doit être entendue: non pas comme une possibilité idéale à partir de laquelle la réalité ne se produit jamais, mais comme cette possibilité ontologique originelle qui constitue en tant que telle la réalité. En l'occurrence la possibilité ontologique originelle de préhension qui constitue la réalité de la main et finalement le corps lui-même en tant que le Je Peux fondamental que je suis. La possibilité ontologique constitutive de la réalité, nous l'appelons Potentialité." Henry, Généalogie de la psychanalyse. Le commencement perdu, (Paris: P.U.F., 1985) p. 394-395. De nouveau, Henry met en place la condition de possibilité d'un élément essentiel de l'être de l'ego, mais il ne décrit pas la singularité d'un mouvement pratiqué par un ego particulier.

33 L'intérêt que porte Henry, dans La barbarie, pour l'autonégation de la vie qu'il retrouve dans la science et la technique moderne, lui permet d'élaborer de nouveaux aspects à l'être de l'ego. Lorsqu'il écrit: “Une vie qui se nie ellemême, l'autonégation de la vie, tel est l'événement crucial qui détermine la culture moderne en tant que culture scientifique" (113), Henry décrit encore une fois un événement propre à l'être de l'ego, au même titre que l'autotransformation et l'autodéveloppement de la vie que nous retrouvons également dans La barbarie. Ce sont des catégories propres à l'être de l'ego. Même si Henry aborde la question de l'art à partir de la figure de Kandinsky, il n'en demeure pas moins que son analyse le mène à décrire le phénomène de l'art en tant que "mode de la vie" ou de l'être: "L'art en vérité est un mode de la vie et pour cette raison, éventuellement, un mode de vie. Il faut donc demander plutôt: comment la vie est-elle présente dans l'art autrement que dans l'existence ordinaire? La réponse, qui justifie l'art ou plutôt qui le désigne comme l'une des activités les plus hautes de l'homme, est celle-ci: la vie est présente dans l'art selon son essence propre. Quelle est l'essence de la vie? Non pas seulement l'épreuve de soi mais, comme sa conséquence immédiate, l'accroissement de soi. S'éprouver soi-même, à la façon de la vie, c'est venir en soi, entrer en possession de son être propre, s'accroître de soi en effet, être affecté d'un "plus" qui est le "plus de soi-même." Voir l'invisible: Sur Kandinsky (Paris: P.U.F., 2005) 209.

35 Dans l'avant-propos à cet ouvrage, Henry décrit explicitement la tâche de la phénoménologie matérielle en tant que recherche de l'essence de la phénoménalité pure. Il écrit: “C'est une vie phénoménologique en ce sens radical que la vie définit l'essence de la phénoménalité pure et par suite de l'être pour autant que l'être est coextensif au phénomène et se fonde sur 
lui." Henry, Phénoménologie matérielle (Paris: P.U.F., 1990) 7. Malgré le fait que Henry privilégie maintenant la vie au dépend de l'être, la recherche demeure la même: établir une description des principaux éléments qui forment l'ego ou le Soi.

36 Rudolf Bernet, "Christianisme et phénoménologie," in Michel Henry: l'épreuve de la vie, dir. A. David et J. Greisch (Paris: Cerf, 2000) 199. Voir l'article de Jean Leclercq au sujet de cet article de R. Bernet: "La provenance de la chair: le souci henryen de la contingence," Studia Phaenomenologica IX (2009): 303-314.

37 Jean, "De l'expérience métaphysique d'autrui à l'intersubjectivité en première personne," 69.

38 Henry, C'est moi la vérité, 313.

39 Henry, C'est moi la vérité, 313.

40 Henry, C'est moi la vérité, 313.

41 Pour une analyse complète de l'identité fonctionnelle et des conséquences sur la vie de l'individu se référer à l'ouvrage de Raphaël Gély, Rôles, action sociale et vie subjective (Bruxelles: P.I.E. Peter Lang, 2007)

Journal of French and Francophone Philosophy | Revue de la philosophie française et de langue française Vol XX, No 2 (2012) | http://www.jffp.org | DOI 10.5195/jffp.2012.521 